\title{
Improved threshold of buried heterostructure InAs/GaInAsP quantum dot lasers
}

Cite as: J. Appl. Phys. 109, 083104 (2011); https://doi.org/10.1063/1.35744406

Submitted: 25 January 2011 • Accepted: 11 March 2011 • Published Online: 18 April 2011

D. Franke, M. Moehrle, A. Sigmund, et al.

\section{ARTICLES YOU MAY BE INTERESTED IN}

Temperature-stable operation of a quantum dot semiconductor disk laser Applied Physics Letters 93, 051104 (2008); https://doi.org/10.1063/1.2968137

Fast gain and phase recovery of semiconductor optical amplifiers based on submonolayer quantum dots

Applied Physics Letters 107, 201102 (2015); https://doi.org/10.1063/1.4935792

Formation of InAs/InGaAsP quantum-dashes on InP(001)

Applied Physics Letters 95, 203105 (2009); https://doi.org/10.1063/1.3265733

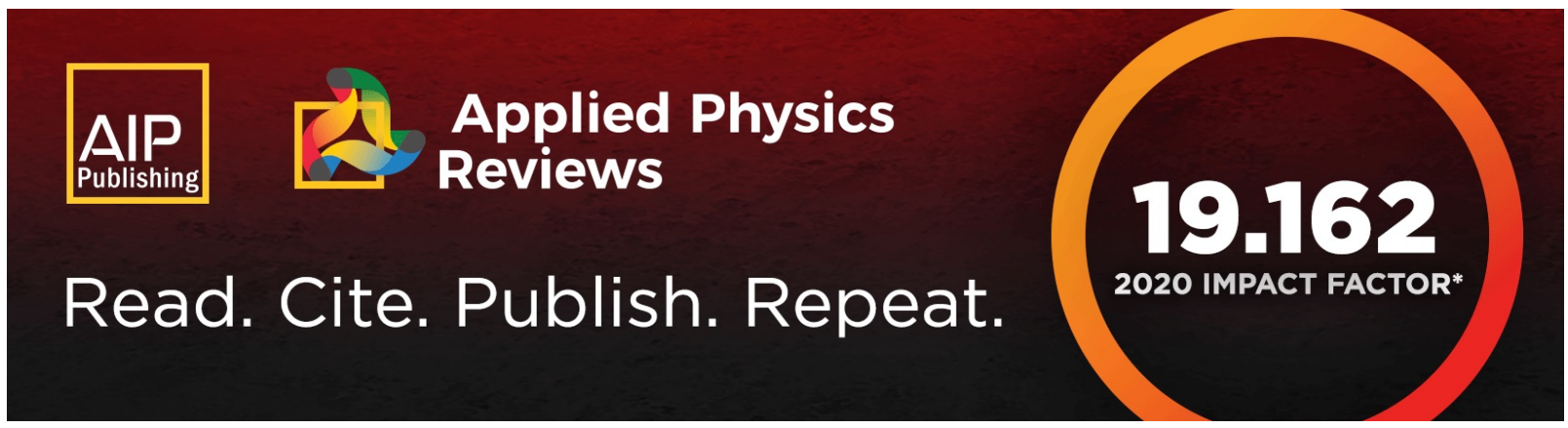




\title{
Improved threshold of buried heterostructure InAs/GalnAsP quantum dot lasers
}

\author{
D. Franke, ${ }^{1}$ M. Moehrle, ${ }^{1}$ A. Sigmund, ${ }^{1}$ H. Kuenzel,,${ }^{1, a)}$ U. W. Pohl,${ }^{2}$ and D. Bimberg ${ }^{2}$ \\ ${ }^{1}$ Fraunhofer Institute for Telecommunications, Heinrich-Hertz-Institut, Einsteinufer 37, 10587 Berlin, \\ Germany \\ ${ }^{2}$ Institut für Festkörperphysik, Technische Universität Berlin, Hardenbergstr. 36, 10623 Berlin, Germany
}

(Received 25 January 2011; accepted 11 March 2011; published online 18 April 2011)

\begin{abstract}
The parameters for reducing the threshold current density of InAs/InGaAsP/InP quantum-dot (QD) lasers suitable for high temperature operation are studied. The structures were grown using metalorganic vapor phase epitaxy. Increasing the number of QD layers leads to a substantial improvement of the optical confinement and a markedly reduced threshold per dot layer in broad area devices. A reduction of the spacer thickness between the QD layers was not found to significantly affect device characteristics. Depending upon the device length, an optimum number of QD layers was deduced. Based upon optimized QD stacks, buried-heterostructure lasers with a medium device length emitting at $1.5 \mu \mathrm{m}$ were fabricated. Laterally single-mode devices show promising low threshold currents near $10 \mathrm{~mA}$ and good thermal stability with a characteristic temperature of $65 \mathrm{~K}$ up to $90^{\circ} \mathrm{C}$. (C) 2011 American Institute of Physics. [doi:10.1063/1.3574406]
\end{abstract}

\section{INTRODUCTION}

Quantum dot (QD) based gain media are advantageous for semiconductor lasers due to the modified electronic density of states which strongly differs from that of higher dimensional systems. ${ }^{1}$ QD lasers based on GaAs demonstrated improved characteristics compared to their quantum well (QW) counterparts, such as reduced threshold current density and improved temperature stability. ${ }^{2,3}$ Devices based on InP using InAs QDs embedded in an InGaAsP matrix are presently less well developed. The best performance reported to date in terms of threshold current, internal efficiency, and characteristic temperature was achieved with structures grown by molecular beam epitaxy (MBE). ${ }^{4,5}$ InAs QDs grown by MBE usually feature a lateral elongation aligned along [011], referred to as quantum dashes. ${ }^{6,7}$ In contrast, dots grown by metalorganic vapor phase epitaxy (MOVPE) were found to be in-plane symmetric. ${ }^{8-10}$ Such dots form in a wide range of MOVPE growth conditions. Dashes like those found in MBE are only formed by applying unusual growth parameters in terms of growth temperature and/or indium flux. ${ }^{11}$ Recently, high quality and thermally stable InAs/InGaAsP/InP QD structures emitting at $1.55 \mu \mathrm{m}$ were grown by MOVPE. ${ }^{12}$ These thermally stable QDs are promising for fabricating QD lasers with characteristics comparable to their MBE-grown counterparts. ${ }^{13}$

A single QD layer generally has a lower amount of active medium as compared to a single QW layer, leading to lower gain saturation levels and output power for QD lasers compared to QW devices with the same number of active layers. The fraction of emitting QD material in the waveguide, i.e., the optical confinement, can be enhanced by increasing the number of QD layers and by reducing the thickness of the InGaAsP matrix spacer-layers. In this contri-

\footnotetext{
a) Author to whom correspondence should be addressed. Electronic mail: harald.kuenzel@hhi.fraunhofer.de.
}

bution, we present the effect of these parameters on the performance of QD broad-area lasers and demonstrate an improved status achieved in corresponding buried heterostructure $(\mathrm{BH})$ devices.

\section{CONDITIONS FOR QD GROWTH}

QD samples were grown by low pressure MOVPE using conventional sources. The formation of QD structures requires flat surfaces. We achieved two-dimensional lowtemperature growth $\left(T_{g} \sim 500{ }^{\circ} \mathrm{C}\right)$ of GaInAsP layers with a bandgap equivalent to $1.15-1.20 \mu \mathrm{m}$ emission wavelength (Q1.15-1.20). These quaternary layers were lattice-matched to InP and were employed as waveguide and matrix materials in our laser structures. InAs QD formation in the range $470-510^{\circ} \mathrm{C}$ leads to dot densities between $5 \times 10^{10} \mathrm{~cm}^{-2}$ and $1.7 \times 10^{10} \mathrm{~cm}^{-2}$, respectively (not shown here). In this study we applied InAs deposition at $500{ }^{\circ} \mathrm{C}$, yielding $2.5 \times 10^{10} \mathrm{~cm}^{-2}$ QDs per layer. The structures contained up to 13 stacked QD layers with typically $30 \mathrm{~nm}$ thick latticematched Q(1.15-1.20) spacer layers. Stacks incorporating more than 13 QD layers yielded inferior material quality. The surface morphology degraded due to strain relief by the formation of dislocation networks.

\section{EXPERIMENTAL RESULTS}

The optical quality of structures containing 3 to $13 \mathrm{QD}$ layers was found to be identical as assessed from high excitation density $300 \mathrm{~K}$ photoluminescence (PL) measurements. Only a structure with a single QD layer showed a sub-linear increase for excitation densities above $1 \mathrm{~kW} \mathrm{~cm}^{-2}$ indicating some saturation. ${ }^{12}$ For all structures with three or more QD layers a linear intensity increase was observed with increasing excitation density within the measured range up to $6 \mathrm{~kW} \mathrm{~cm}^{-2}$. The majority of the excited carriers recombine via quantum dot states as concluded from PL data. Only a 
small fraction of the carriers recombines in the quaternary matrix material as indicated by the weak PL feature at 1150 $\mathrm{nm}$ in Fig. 1. No additional broadening of the PL is found as the number of QD layers is increased, indicating good interlayer uniformity. The large full width at half maximum of the QD emission of $110 \mathrm{meV}$ originates from intralayer QD size fluctuations. Fluctuations of the QD height are the main reason for this, since the height is markedly smaller than the lateral dimensions (not shown here) and thus essentially determines the quantization energy.

\section{A. Fabrication of broad area QD lasers}

To study the effect of the number of QD layers on the optical confinement we processed broad-area lasers using structures with $3,5,7$, and 13 QD layers grown at $500^{\circ} \mathrm{C}$. The $50 \mathrm{~nm} \mathrm{Q}(1.15)$ undoped waveguide layers with $2 \times 10^{17}$ $\mathrm{cm}^{-3} \mathrm{n}$-doped ( $\mathrm{Si}$ ) and p-doped ( $\left.\mathrm{Zn}\right) \mathrm{InP}$ cladding layers, and a $200 \mathrm{~nm}$ InGaAs:Zn contact layer doped to a level of $1 \times 10^{19} \mathrm{~cm}^{-3}$ were used. The temperature for growth below the active layers was $640^{\circ} \mathrm{C}$, while it was lowered to $620^{\circ} \mathrm{C}$ for layers deposited after the QD stacks to reduce potential intermixing. The growth of the whole structure was completed by an in situ $\mathrm{Zn}$ indiffusion to increase the p-doping level to $8 \times 10^{19} \mathrm{~cm}^{-3}$ near the surface.

Broad-area lasers $50 \mu \mathrm{m}$ in width, along with seven QD layers were processed, mounted, and operated in continuous wave (cw) mode. From the length dependence a threshold per dot layer of $100 \mathrm{~A} \mathrm{~cm}^{-2}$ for an infinite length at room temperature was deduced. Furthermore, a characteristic temperature $T_{0} \sim 65 \mathrm{~K}$, an absorption coefficient $\alpha=5 \mathrm{~cm}^{-1}$, and an internal quantum efficiency $\eta=0.65$ were achieved.

\section{B. Influence of design parameters}

Threshold data of broad-area devices with $50 \mu \mathrm{m}$ ridge width and a varied number of QD layers were recorded on a

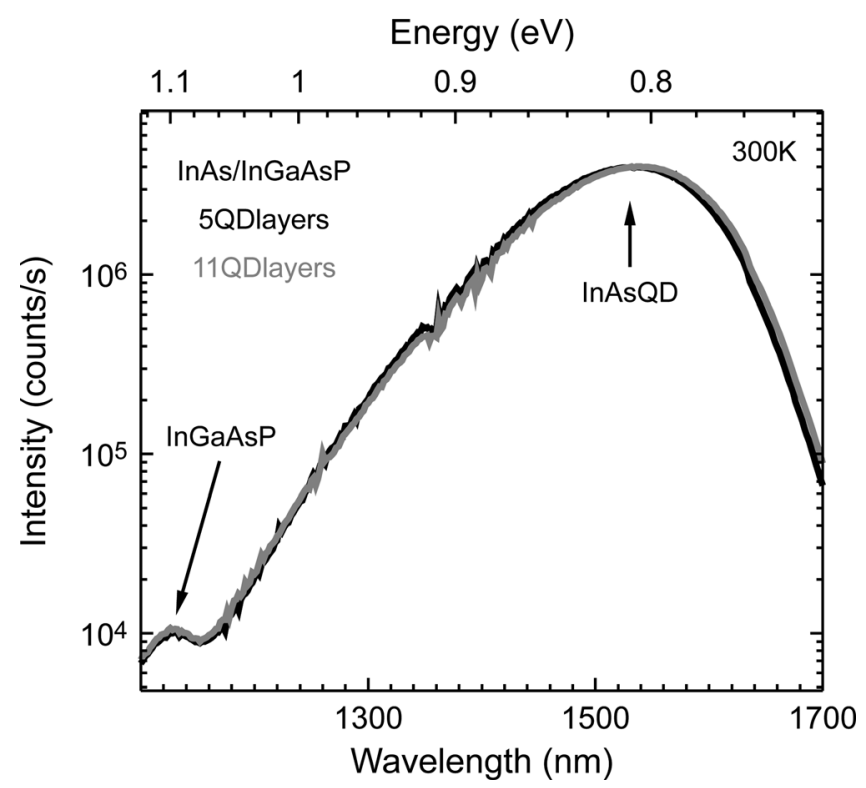

FIG. 1. Room-temperature photoluminescence spectra of five-fold (black) and eleven-fold (gray) stacked InAs/InGaAsP QD layers nonresonantly excited with $500 \mathrm{~W} \mathrm{~cm}^{-2}$. chip in pulsed mode for simplicity. The results are summarized in Fig. 2. The data depict the threshold per dot layer versus the inverse cavity length for devices with different numbers of QD layers. A marked influence of the number of QD layers on the threshold current density per dot layer is obvious. From the exponential fits, a decrease of the threshold per dot layer at infinite length $L$ from 210 to $90 \mathrm{~A} \mathrm{~cm}^{-2}$ is found as the number of dot layers is increased from three to seven. The slope of the $\ln (J)$ versus $1 / L$ dependence allows us to determine the optimum resonator length. ${ }^{14}$ These values, marked by arrows in Fig. 2, decrease from $640 \mu \mathrm{m}$ for three QD layers to $370 \mu \mathrm{m}$ for seven QD layers. By plotting the threshold current density at the optimum resonator length versus the inverse number of QD layers a transparency current density of $77 \mathrm{~A} \mathrm{~cm}^{-2}$ and a gain constant of $9 \mathrm{~A} \mathrm{~cm}^{-1}$ were deduced. Based upon these results, a further improvement of the optimum length can mainly be achieved by further increasing the number of QD layers incorporated in the active core of the laser structure. Devices with up to 13 QD layers still show some minor improvement of the threshold per dot layer at infinite length to $70 \mathrm{~A} \mathrm{~cm}^{-2}$. Additionally, the slope of the relation $\ln (J)$ versus $1 / L$ shows some minor improvement.

The decrease of threshold per dot layer at infinite length is accompanied by a linear increase of the absorption coefficient with the number of dot layers. The values increased from 5.7 (five QD layers) to $11.5 \mathrm{~cm}^{-1}$ (13 QD layers). By comparing these results with the data of a reference structure containing eight QWs, given by the gray line in Fig. 2, we conclude: i) the threshold per dot layer at infinite length of these devices is virtually identical with comparable QW structures, however ii) the threshold for short cavity QD lasers is still inferior due to the smaller slope of the $\ln (J)$ versus $1 / L$ dependence as is also found for quantum dash lasers grown by MBE. ${ }^{4}$ For $200 \mu \mathrm{m}$ long devices the difference in

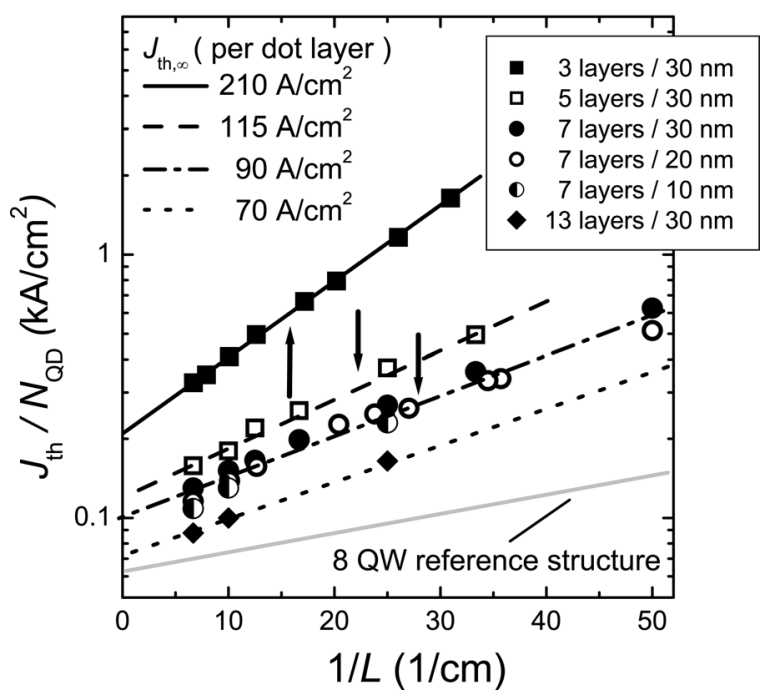

FIG. 2. Device-length dependence of the threshold current density per dot layer of broad-area QD lasers (width $=50 \mu \mathrm{m}$ ) emitting at $1.55 \mu \mathrm{m}$. The devices contain various numbers of QD layers and thicknesses of spacers between the QD layers as indicated in the figure. Arrows mark optimum cavity lengths and the gray line refers to a similar structure with eight quantum wells. 
threshold amounts to a factor of 3 as deduced from Fig. 2 for $1 / L=50 \mathrm{~cm}^{-1}$. To attain similar values as those of the $\mathrm{QW}$ structures an increase of the density of the QD layers might be a viable way. Alternatively, an increase of the dot density per QD layer may also lead to quantum-dash formation for MOVPE growth due to the required change in deposition conditions. ${ }^{15}$

We kept the growth parameters constant and fabricated a device with seven QD layers, where the spacer thickness was varied between 40 and $10 \mathrm{~nm}$. The data included in Fig. 2 show virtually no difference in performance within the scatter of the data. This indicates that a more dense stacking of QD layers does not lead to a significant improvement of threshold. Further reduction towards a $10 \mathrm{~nm}$ spacer did not degrade laser performance and gives practically identical laser characteristics as with 20 or $30 \mathrm{~nm}$ spacers. In contrast, an increase in QD-layer separation to $40 \mathrm{~nm}$ leads to an increase of the threshold per dot layer at infinite length from 90 to $135 \mathrm{~A} \mathrm{~cm}^{-2}$ (data not included in Fig. 2).

For the practical design of lasers with improved injection current, as is necessary for stable high temperature operation, the overall threshold current is of concern. According to Ref. 14 (Eq. 17), the overall threshold in conventional multiple quantum well lasers is mainly dominated by its dependence upon the number of quantum wells. This dependence comprises both; a factor being linearly dependent and a factor decreasing exponentially with the number of quantum wells. As a result, a minimum overall threshold in dependence of the number of quantum wells can be deduced for a fixed laser length. We have applied the same evaluation procedure to our QD lasers. The corresponding results are depicted in Fig. 3 for three different laser lengths. Besides the expected appearance of a minimum threshold, it can clearly be seen in Fig. 3 that due to the additional dependence on laser length, the number of QD layers suitable for a minimum threshold increases for finite lengths. For lasers of

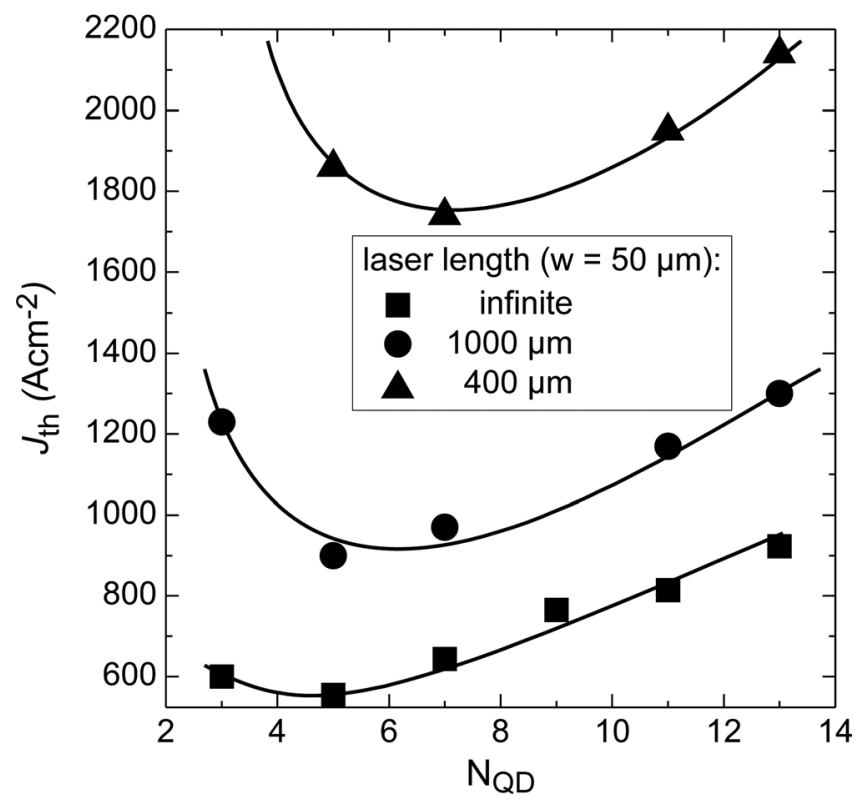

FIG. 3. Dependence of the threshold current density on the number of QD layers for broad-area lasers of different lengths. infinite length (extrapolated) the optimum number of QD layers is five. This value increases to six layers for very long lasers $(L=1000 \mu \mathrm{m})$ and to seven layers for devices with a medium length $(L=400 \mu \mathrm{m})$.

\section{Buried heterostructure QD lasers}

To demonstrate the achieved capability of InAs/ InGaAsP QD structures grown using MOVPE, we fabricated $1.4 \mu \mathrm{m}$ wide laterally single mode BH lasers. We applied growth conditions also used for the broad-area devices and incorporated seven QD layers as an optimum for medium length devices. The current flow to the $1.4 \mu \mathrm{m}$ wide laser ridge was restricted by $\mathrm{p} / \mathrm{n}$-blocking. A scanning electron micrograph of the cleavage plane showing the $1.4 \mu \mathrm{m}$ wide ridge with the active QD region and the $\mathrm{p} / \mathrm{n}$ blocking layers is given in Fig. 4(a). The emission of these devices was blueshifted by typically $50-100 \mathrm{~nm}$ with respect to that of the unprocessed structure. The shift originates from the regrowth of the blocking and contact layers and may be balanced in structures thermally optimized with respect to the regrowth process. The effect may also be suppressed by a decrease of the regrowth temperature and a slightly higher growth temperature of the QD layers to obtain a higher thermal stability of the QDs. ${ }^{13}$ BH-type QD laser devices were prepared by cleaving the structures to lengths between 300 and $400 \mu \mathrm{m}$. Pulsed light output versus current $L I$-characteristics of these uncoated devices given in Fig. 4(b) showed typical threshold currents between 10 and $20 \mathrm{~mA}$ at room temperature. Devices were operated up to $90^{\circ} \mathrm{C}$ with the threshold increasing only up to $30 \mathrm{~mA}$ in agreement with a characteristic temperature, $T_{0}$, of $65 \mathrm{~K}$ similar to broad-area devices. An output power per facet of $10 \mathrm{~mW}$ at $200 \mathrm{~mA}$ drive current of the unmounted lasers was achieved. Gain spectra were deduced from $\mathrm{cw}$ emission spectra recorded close to threshold as depicted in Fig. 5(a). By analyzing the longitudinal spectral mode behavior using the Hakki-Paoli method, a $80 \mathrm{~nm}$ wide gain spectrum peaking at $1490 \mathrm{~nm}$ was extracted for a 330 $\mu \mathrm{m}$ long device driven at $10 \mathrm{~mA}$, cf. Fig. 5(b). A peak net gain of $30 \mathrm{~cm}^{-1}$ corresponding to a value per dot layer close to $5 \mathrm{~cm}^{-1}$ was determined. This compares with a net gain at
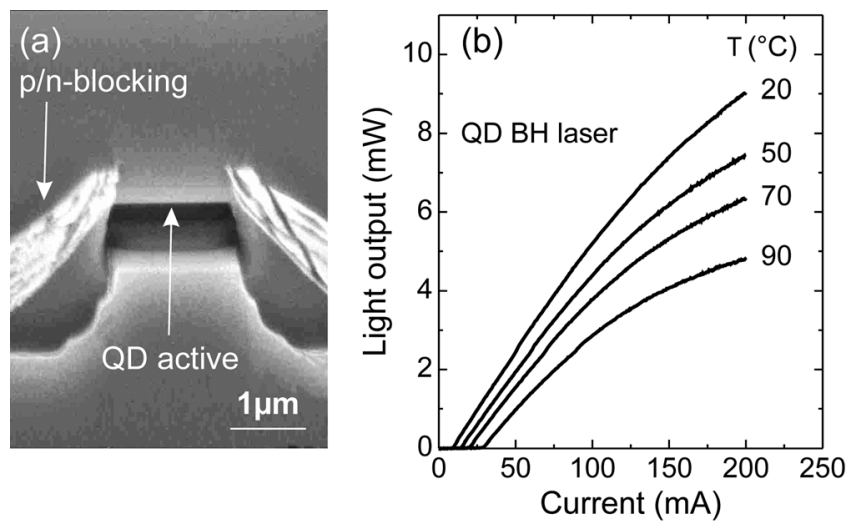

FIG. 4. BH-type laser containing seven InAs QD layers and a Q(1.15) matrix. (a) Scanning electron micrograph of the cleavage plane of the device showing the active QD region and p/n-blocking layers. (b) Static $L I$-characteristics for different operation temperatures of a $330 \mu \mathrm{m}$ long and $1.4 \mu \mathrm{m}$ wide device in pulsed mode. 


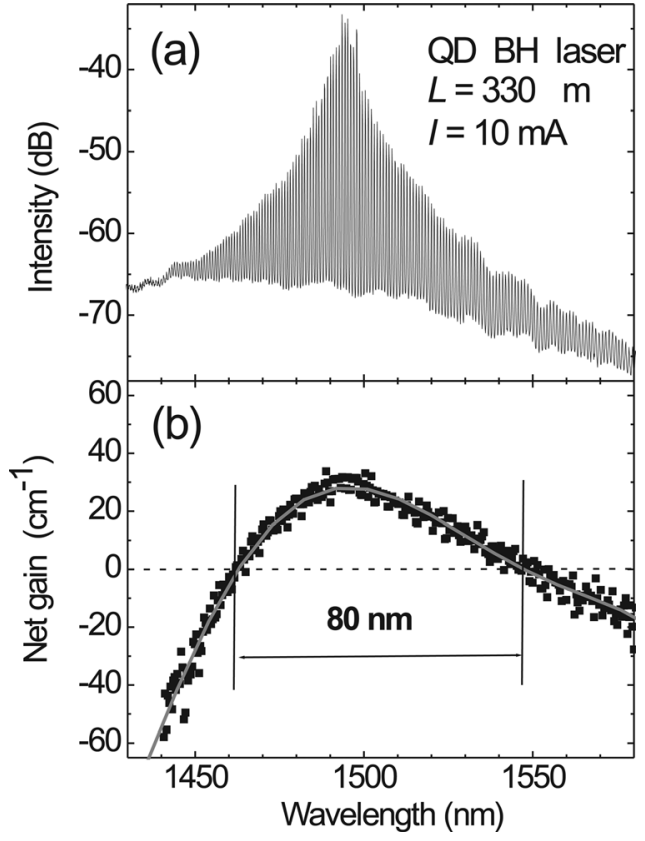

FIG. 5. (a) Mode spectrum of the BH QD laser shown in Fig. 4 taken slightly below threshold at a $10 \mathrm{~mA}$ injection currrent in cw-mode. (b) Wavelength dependence of the net gain as deduced from the data of (a) using the Hakki-Paoli method.

threshold of $19 \mathrm{~cm}^{-1}$ in a MBE grown buried ridge stripe device with six QD layers and $40 \mathrm{~nm}$ spacers $^{5}$ demonstrating the competitiveness of our MOVPE approach.

\section{CONCLUSIONS}

To conclude, the main parameters for reducing the threshold of MOVPE-grown InP-based QD lasers have been presented. Only the number of QD layers was found to markedly affect the optical confinement and thus the laser threshold. Optimum performance was obtained for devices comprising seven QD layers. First structures for BH-type device fabrication operating laterally single mode near $1.5 \mu \mathrm{m}$ showed a promising low threshold near $10 \mathrm{~mA}$ without facet coating, a high net gain of $30 \mathrm{~cm}^{-1}$, and a good thermal stability with $T_{0}=65 \mathrm{~K}$ up to $90^{\circ} \mathrm{C}$.

\section{ACKNOWLEDGMENTS}

Part of the work was funded by the German Research Foundation DFG within the SFB787 project A3. The authors thank W.-D. Molzow for expert help in laser processing and characterization.

${ }^{1}$ M. Asada, Y. Miyamoto, and Y. Suematsu, IEEE J. Quantum Electron. QE-22, 1915 (1986).

${ }^{2}$ D. Bimberg, J. Phys. D: Appl. Phys. 38, 2055 (2005).

${ }^{3}$ O. B. Shchekin, J. Ahn, and D. G. Deppe, Electron. Lett. 38, 712 (2002).

${ }^{4}$ J. P. Reithmaier, A. Somers, S. Deubert, R. Schwertberger, W. Kaiser, A. Forchel, M. Calligaro, P. Resneau, O. Parillaud, S. Bansropun, M. Krakowski, R. Alizon, D. Hadass, A. Bilenca, H. Dery, V. Mikhelashvili, G. Eisenstein, M. Gioannini, I. Montrosset, T. W. Berg, M. van der Poel, J. Mork, and B. Tromborg, J. Phys. D: Appl. Phys. 38, 2088 (2005).

${ }^{5}$ F. Lelarge, B. Rousseau, B. Dagens, F. Poingt, F. Pommerau, and A. Accard, IEEE Photon. Technol. Lett. 17, 1369 (2005).

${ }^{6}$ O. Bierwagen and W. T. Masselink, Appl. Phys. Lett. 86, 113110 (2005).

${ }^{7}$ G. Saint-Girons, A. Michon, I. Sagnes, G. Beaudoin, and G. Patriarche, Phys. Rev. B 74, 245305 (2006).

${ }^{8}$ J. W. Jang, S. H. Pyun, S. H. Lee, I. C. Lee, W. G. Jeong, R. Stevenson, P. D. Dapkus, N. J. Kim, M. S. Hwang, and D. Lee, Appl. Phys. Lett. 85, 3675 (2004).

${ }^{9}$ K. Kawaguchi, M. Ekawa, A. Kuramata, T. Akiyama, H. Ebe, M. Sugawara, and Y. Arakawa, Appl. Phys. Lett. 85, 4331 (2004).

${ }^{10}$ S. Anantathanasarn, R. Nötzel, P. J. van Veldhoven, T. J. Eijkemans, and J. H. Wolter, J. Appl. Phys. 98, 013503 (2005).

${ }^{11}$ A. Lenz, F. Genz, H. Eisele, L. Ivanova, R. Timm, D. Franke, H. Künzel, U. W. Pohl, and M. Dähne, Appl. Phys. Lett. 95, 203105 (2009).

${ }^{12}$ D. Franke, P. Harde, J. Boettcher, M. Moehrle, A. Sigmund, and H. Kuenzel, Proc. 19th International Conference on InP and Related Materials, Matsue, 2007, IEEE Catalog \# 07CH37859C, ISBN \# 1-4244-0874-1, p. 559-562, 2007.

${ }^{13}$ D. Franke, M. Moehrle, J. Boettcher, P. Harde, A. Sigmund, and H. Kuenzel, Appl. Phys. Lett. 91, 081117 (2007).

${ }^{14}$ M. Rosenzweig, M. Moehrle, H. Dueser, and H. Venghaus, IEEE J. Quantum Electron. 27, 1804 (1991).

${ }^{15}$ D. Franke, J. Kreissl, W. Rehbein, H. Künzel, U. W. Pohl, and D. Bimberg, Appl. Phys. Express 4, 014101 (2011). 